\title{
Für jede Indikation das ideale Komposit
}

Mit den Venus Kompositen von Heraeus Kulzer bekommt der Begriff „Universalkomposit” für Zahnärzte eine neue Bedeutung, denn die Komposite können nach persönlichen Vorlieben ausgewählt und kombiniert werden. Die Behandler decken mit ihrer Wahl nahezu jede Indikation ab und gewinnen so mehr Flexibilität und bequemere Arbeitsabläufe. Dank seiner festen, minimal klebrigen Konsistenz ist Venus Diamond gut formbar. Mit seiner cremigen und gleichzeitig kaum klebrigen Konsistenz bietet Venus Pearl eine gute Modellierbarkeit. Beide Komposite basieren auf der patentierten TCDMatrix und eignen sich sowohl für Einschicht- wie auch für Mehrschichtverfahren. Bei kleineren Kavitäten, für Zahnhalsfüllungen oder als Baseliner in Kombination mit Kompositen wie Venus Diamond oder Venus Pearl kann das höherviskose Venus Diamond Flow genutzt werden.

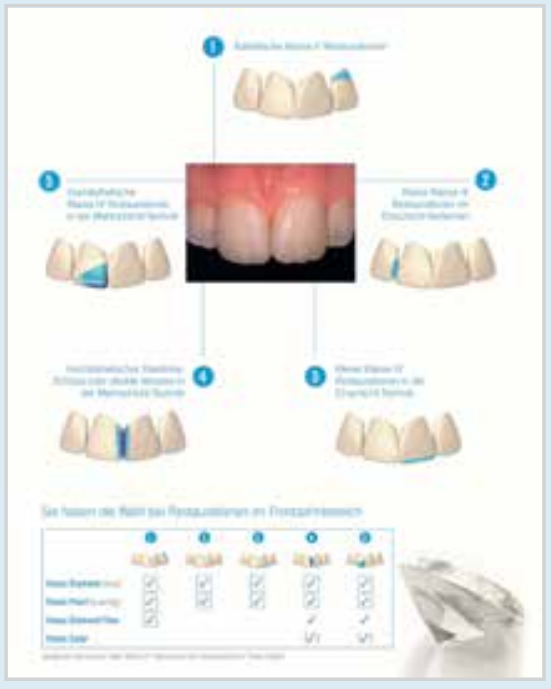

Als weiteres Mitglied in der Kompositfamilie ist Venus Bulk Fill das fließfähige, schrumpfkraftreduzierte Komposit für Restaurationen im Seitenzahnbereich. Das Material ermöglicht einfache Füllun- gen in Inkrementen bis $4 \mathrm{~mm}$ bei Kavitäten der Klasse I und II. Venus Color ist die ideale Ergänzung zu den genannten Kompositen, wenn Farbe ins Spiel kommt, z. B. wenn Risse im Zahnschmelz, Verfärbungen von Fissuren oder weiße Flecken nachzubilden sind.

Um die Auswahl und Kombination der Produkte und Farben für Zahnärzte noch einfacher zu gestalten, hat Heraeus Kulzer Venus Mix \& Match-Angebote zusammengestellt. So können Behandler nach ihren persönlichen Vorlieben auswählen und umfassend von diesem Angebot profitieren.

Nach einer Pressemitteilung der Heraeus Kulzer GmbH, Hanau Internet: www.heraeus-kulzer.com 